\title{
DIABETES MELLITUS GESTACIONAL: UMA VISÃO GERAL
}

\section{ARTIGO DE REVISÃO}

PAIXÃO, Carmen Scheyla Dettmann ${ }^{1}$

ZORZAL, Juliano Kacio ${ }^{2}$

PAIXÃO, Carmen Scheyla Dettmann. ZORZAL, Juliano Kacio. Diabetes mellitus gestacional: Uma visão geral. Revista Científica Multidisciplinar Núcleo do Conhecimento. Ano 05, Ed. 10, Vol. 04, pp. 05-20. Outubro de 2020. ISSN: 24480959, Link de acesso: https://www.nucleodoconhecimento.com.br/saude/diabetesmellitus-gestacional

\section{RESUMO}

O principal objetivo deste artigo é fundamentar ações sobre a Diabetes Mellitus através do assistencialismo farmacêutico durante o período gestacional. Por meio da análise da literatura farmacêutica e também através do estudo que versam sobre a Diabetes Mellitus durante o período gestacional. O principal papel do assistencialismo farmacêutico está em conceber espaços para o desenvolvimento de uma assistência preocupada com a saúde das pacientes no período gestacional. A metodologia dessa pesquisa foi baseada na base documental, com análise da literatura farmacêutica que normalizam as regras da Farmácia Brasileira. Dentre as literaturas citamos Tombini, Kitzmiller; Davidson, Bertini e demais autores que fomentam a literatura brasileira. O desfecho do presente trabalho consiste na identificação dos efeitos e das causas da Diabetes Mellitus durante o período gestacional e por fim, procurando orientar e esclarecer algumas atitudes com o intuito de orientar melhor as gestantes durante 0 período da gravidez.

\footnotetext{
${ }^{1}$ Acadêmica Do Curso Superior De Farmácia.

${ }^{2}$ Orientador. Especialização em Educação Profissional e Tecnológica. Graduação em Farmácia. Graduação em Ciências/Matemática.
} 
Palavras-chaves: DMG, fatores de risco, cuidados.

\section{INTRODUÇÃO}

A disglicemia, ou seja, a alteração da glicemia é atualmente uma das maiores preocupações da medicina enquanto a mulher se encontra no período gestacional, tornando um dos fatores de risco na gestação. Desta forma, a diminuição da sensibilização à insulina fica evidenciada em diferentes estágios da vida, mas é considerada como uma preocupação enquanto no período da gravidez.

Assim, uma difícil adequação endócrino-metabólica acontece durante a gravidez, envolvendo alterações da insulina, assim como um acréscimo da resposta e da massa das células beta e também pequena alta da glicemia principalmente ao findar das refeições, mudanças nas taxas circulantes fosfolipídicas, ácidos graxos livres, triglicérides e do colesterol.

O desajuste metabólico mais regular durante a gestação é o diabetes gestacional e sua apresentação mais predominante é determinada como uma modificação da glicemia de qualquer nível, descoberta no período de gravidez.

Ao expressar o Diabetes Mellitus Gestacional é preciso definir os níveis de intolerância aos carboidratos, resultados em elevação da glicose no sangue de magnitudes variáveis, com origem ou diagnóstico durante a gestação. Grandes modificações ocorrem na geração de energia e ajuntamento de gordura durante a gravidez normal. Esse depósito de gordura se identifica especialmente na primeira metade da gestação, enquanto no seu final há aumento no gasto metabólico da mãe.

Enfim, é essencial que a mãe seja avaliada todo o período gestacional, observando o estado e os níveis glicêmicos. Desta forma, é proposto o controle de peso, dietas e exercícios e uma glicemia de jejum realizada periodicamente. 


\section{ETIOLOGIA}

Diabete Mellitus (DM) é uma patologia metabólica, descrita como elevação do volume da glicose no sangue oriunda de deficiência na geração e/ou na ação da insulina. Desta conformação, Diabetes Mellitus Gestacional (DMG) é entendida como qualquer classe de intolerância à glicose, com começo ou primeira constatação na gestação.

Este conceito se adota apesar da administração de insulina ou se a conjuntura continua depois do parto e não elimina a possibilidade de a intransigência à glicose ter sucedido na gravidez. As alterações identificadas no metabolismo materno enquanto perdurar a gestação é imprescindível a fim de contemplar as exigências do feto.

Outro quesito importante é a ocorrência da resistência à insulina $(\mathrm{Rl})$ na segunda parte da gestação como desfecho do ajuste fisiológico, permeada pelos hormônios placentários anti-insulínicos, para assegurar o subsídio adequado de glicose ao feto. Contudo, certas gestantes que engravidam com qualquer grau de RI com sobrepeso e síndrome de ovário policístico, esta condição fisiológica de RI será elevado nos tecidos

periféricos.

Simultaneamente, é necessidade fisiológica uma maior produção de insulina, assim como da falta de capacidade do pâncreas em responder a RI fisiológica auxilia o quadro de hiperglicemia de impetuosidade diversificada, acusando o DMG.

Gestantes que já são insulinorresistentes não tem a capacidade de elevar a taxa de eliminação de insulina indispensável para contemplar as necessidades da sua gestação. Essa resistência já está presente na segunda metade da gravidez e eleva progressivamente até o fim da gravidez, quando ela se semelha a intolerância à glicose observada no tipo 2 da diabetes. Assim, a resistência à insulina ajuda as demandas metabólicas do feto (maior oferta de glicose) e é resultado dos hormônios placentários circulantes. 
A imperfeição metabólica nas grávidas com DMG é a sua falta de capacidade de produzir insulina em níveis necessários à necessidade que é máxima no 3 o trimestre, levando à elevação da concentração de glicose pós-prandial deficiente para acarretar sintomas, mas capaz de estabelecer efeitos adversos no feto (hipoglicemia neonatal e macrossomia) pelo demasiado de glicose transplacentário.

\subsection{IMPORTÂNCIA DO DIAGNÓSTICO}

Tal como é verificado em exames para detecção da diabetes, sua causa exata ainda não foi descoberta e os estudos levam a crer em várias hipóteses. Contudo, de acordo com estudos da American Diabetes Association (TOMBINI, 2002) elencam algumas hipóteses:

- Hormonal - Kitzmiller; Davidson (2001) definem que enquanto o período da gravidez perdurar a placenta produz quantidades de hormônios em grande escala e como a progesterona, o estrogênio e a somatomamotrofina coriônica humana são imprescindíveis para o desenvolvimento fetal, tem potencial para interferir na ação da insulina no organismo materno, atuando como antagonista da atividade da insulina, causando elevação na resistência à insulina nos últimos dois trimestres de gestação;

- Genética - Tombini (2002) declara a diabete gestacional como aquela qualificada pela resistência à insulina análoga ao que acontece ao diabetes tipo 2. Nesse contexto, as mulheres que manifestam diabetes durante o período gestacional têm maior viabilidade de apresentar o diabetes tipo 2.

- Obesidade - Tombini (2002) esse tipo de diabetes é mais corrente em mulheres em que a obesidade faz parte de suas vidas. A obesidade pode desprender tanto o diabetes gestacional assim como o diabetes mellitus tipo 2 .

O Ministério da Saúde recomendou no Manual de Gestação de Alto Risco de 2012 o uso de causas clínicas de risco para DMG, agregadas a uma glicemia de jejum no começo da gravidez (anterior há 20 semanas ou o mais rápido possível), para a investigação de DMG. 
O estudo HAPO-2008 (IADPSG, 2010) serve-se do teste oral de tolerância à glicose de $75 \mathrm{~g}$ e de $2 \mathrm{~h}$ (TOTG-75) entre 24-28 semanas sem rastreamento preliminar pelos elementos de risco ou através do teste oral de tolerância à glicose de $50 \mathrm{~g}$ de $1 \mathrm{~h}$ (TOTG-50).

O TOTG-50 pretende ser diagnóstico e exige dieta livre três dias antes do exame e as taxas s anormais são jejum $92 \mathrm{mg} / \mathrm{dL}, 1$ hora $180 \mathrm{mg} / \mathrm{dL}$ e de 2 horas $153 \mathrm{mg} / \mathrm{dL}$. Basta uma taxa alterada para o teste ser conceituado como positivo. E se o jejum for $126 \mathrm{mg} / \mathrm{dL}$ a diabetes é considerada pré-gestacional.

A glicose da mãe transfere-se para a porção fetal por dilatação facilitada e, na ocasião se a mãe for hiperglicêmica, o feto, por sua vez, também será hiperglicêmico. Tal como o pâncreas do feto está gerado e ativo a partir da 10ª semana, acontecerá resposta a esta estimulação, com consequente hiperinsulinemia do feto.

A insulina que é um hormônio anabolizante que, ligada ao substrato da energia hiperglicêmica, estabelecerá a macrossomia do feto juntamente com todas as suas consequências, entre elas, o risco aumentado de toco traumatismos.

Uma outra desordem da hiperglicemia se dá no acréscimo da diurese fetal, levando ao polidrâmnio, desordem que auxilia a ruptura prematura de membranas e a prematuridade. A hiperglicemia do ventre intrauterino está ligada á elevação de radicais livres de oxigênio, incumbidos pela maior sucessão de malformações de feto nesse público. Após a laqueadura do cordão umbilical, o neonato metaboliza ligeiramente a glicose pela sobra na geração de insulina e, como desfecho, apresenta hipoglicemia neonatal.

Uma das principais desordens do feto em gestantes com DMG é a macrossomia7, que está relacionada à obesidade infantil e também ao risco elevado de síndrome metabólica (SM) na fase adulta. Não só a macrossomia, mas bem como o alargamento intrauterino reduzido estão implicados na criação da Síndrome Metabólica de de seus componentes. A elevação da glicose na corrente sanguínea (hiperinsulinemia), também se inclui na geração do surfactante pulmonar, ocasionando retardo no amadurecimento pulmonar 
fetal e, consequentemente ao risco elevado da síndrome de desconforto respiratório (SDR) no período neonatal.

Desta forma, a elevação da taxa de glicemia materna relaciona-se à maior aglomeração da hemoglobina glicada (HbA1c), que por sua vez, tem maior empatia por oxigênio e beneficia a hipóxia de graus desiguais. O feedback do feto à hipóxia é a elevação na geração de glóbulos vermelhos, apoliglobulia.

A superabundância ou pletora fetal é responsável pelo maior acontecimento de icterícia neonatal, trombose de veia renal e risco elevado de kernicterus. Contudo, os maiores riscos de morbimortalidade estão nesses casos e o morte intrauterina notadamente nas quatro últimas semanas de gestação, é próprio do DMG mal contido e mais comum em fetos com macrossomia 15,16.

\subsection{FISIOPATOLOGIA}

Diabetes Mellitus (DM) representa um aglomerado de distúrbios metabólicos identificados por hiperglicemia consequente à irregularidade insulínica. Tal irregularidade pode ser proveniente da geração pancreática diminuída ou da indevida liberação e/ou da resistência periférica insulínica $(8,9,13)$.

A designação da etiopatogenia da disglicemia beneficia a compreensão da fisiopatologia e oferece o fundamento para certa condução de cada caso nas diversas etapas da vida dos indivíduos. O período da gravidez se caracteriza pelo estado de resistência insulínica. Essa situação, associada à acentuada mudança nos dispositivos de controle da glicemia em relação à aplicabilidade do gasto de glicose pelo embrião e feto, pode colaborar para ocorrência de modificações da glicemia favorecendo o desenvolvimento de DMG.

Hormônios como cortisol, lactogênio placentário, prolactina gerados pela placenta e outros que são elevados devido o período gestacional, podem estimular a diminuição da ação da insulina nos seus receptores e, por fim, uma elevação da geração insulínica nas gestantes saudáveis. 
Tal mecanismo, no entanto, tem chance não ser presenciado nas grávidas que já se encontram com seu poder de geração de insulina no limite. Isto ocorre nas mulheres que têm escassa elevação de geração de insulina e, assim, podem apresentar diabetes durante a gravidez.

As atuais diretrizes da Organização Mundial da Saúde (OMS) e dos fundamentais regulamentos de manejo de DM aconselham que o aumento na taxa de açúcar no sangue, a hiperglicemia primeiramente detectada em qualquer instante da gestação deve ser classificada e caracterizada em DMG ou DM diagnosticado na gestação do inglês Overt Diabetes $(8,9,14,15)$. Divide-se em duas grandes categorias:

- Diabetes Mellitus Gestacional (DMG): constatada pela primeira vez no período da gravidez, com taxas glicêmicas sanguíneas que não completam as especificações diagnósticas para DM.

- Diabetes Mellitus constatado na gravidez (Overt Diabetes): sem diagnose anterior de DM, com hiperglicemia detectada na gravidez e com taxas glicêmicas sanguíneas que atingem as especificações da OMS para a DM na inexistência de gestação.

As manifestações fisiopatológicas do DMG estão associadas às ajeitações metabólicas ocasionadas durante a gravidez. Bertini (2001) define como pela necessidade contínua de ingerir glicose e de aminoácidos importantes para o feto, acrescentando-se as demandas de ácidos graxos e colesterol e às mudanças hormonais, essencialmente as definidas pelo glucagon, glicocorticoides, estrogênios, progesterona e somatotropina coriônica. Conforme Kitzmiller e Davidson (2001) no primeiro trimestre da gestação as mudanças estimuladas por hormônios placentários como a gonadotrofina cariônica humana ( $\mathrm{hCG}$ ) tem rara consequência diretora sobre o metabolismo dos carboidratos. Traçando uma comparação ao desenvolvimento da placenta acontece gradativamente uma elevação na geração de hormônios que antagonizam a ação da insulina, como a progesterona, o estrógeno e principalmente a somatotrofina coriônica humana. Com isso no segundo e no terceiro trimestre da gravidez é visível uma elevação da resistência à insulina, repercutindo em elevação na acumulação desta. 
Identificamos uma demanda na produção de insulina maior que a capacidade de células pancreáticas de produzir insulina, ocorrendo a instalação da diabetes mellitus gestacional. Assim, observam-se na gravidez duas etapas diferenciadas, uma fase anabólica e uma catabólica, a primeira é evidenciada por uma redução da glicemia por superior armazenamento da glicose, enquanto que a segunda por uma redução da glicemia por maior consumo do feto, (SANKOVSKI, 1999). Identificam-se, nesse momento, condições metabólicas na grávida normal caracterizada por um estado limiar de

jejum.

Bertini (2001) também estabelece como uma condição de jejum materno alongado que conduz a um estado metabólico desenfreado de inanição, coagindo ao organismo a se valer de energia, em específico a hidrólise de triglicérides no tecido adiposo. Forçando a investigação de novas fontes de glicose que acaba por gerar novas reações que elevam os corpos cetônicos e os ácidos graxos livres.

\subsection{SINAIS E SINTOMAS}

O diabetes gestacional, assim como os outros tipos de diabetes, pode oferecer desordens, tanto em menor prazo quanto em maior prazo. As grávidas portadoras de DMG são taxadas como grávidas de risco, pois o ultraje constitui altos índices de morbidade, além de propiciar maiores oportunidades para intolerância à glicose, o que pode causar mais riscos de apresentarem DM2 após a gravidez.

As alterações hormonais no começo da gestação incitam vômitos e náuseas em muitas mulheres e nas diabéticas possuidoras de neuropatia autonômica ou gastroparesia, a hipermerese pode ser destrutiva (KITZMILLER; DAVIDSON, 2001).

Os riscos e desordens ainda não são bem elucidados, por essa razão, nos últimos anos, diversas investigações têm sido efetuadas sobre esse tema, inclusive tratando os novos métodos de diagnóstico do DMG, que enfrentaram modificações de 2005 até os dias atuais (FERRARA, 2007; SBD, 2014-2015).

Frequentemente, o diagnóstico de DMG é feito por meio de investigação ativa, com exames que estimulam carga excessiva de glicose, no segundo trimestre da 
gestação. Contudo, nos dias atuais, a sugestão é que se realize a seleção precoce de DMG nas grávidas, logo na primeira consulta de pré-natal, possibilitando, assim, identificar casos de DM preexistentes, que não tem probabilidade ser considerados DMG (SMCD, 2010 apud WEINERT, 2011; IADPSG CONSENSUS PANEL, 2010).

Em sua análise, Simon, Marques e Farhat (2013), asseguram que até o presente, existem muitos conflitos acerca do ponto de corte para a glicemia de jejum na investigação do DMG, e que essa referência varia de acordo com o público pesquisado e o ponto de corte utilizado. Estes autores declaram que não se achou ocorrências de DMG nas pacientes que manifestaram glicemia de jejum menor que $90 \mathrm{mg} / \mathrm{dL}$ no primeiro trimestre ou que não possuíssem fator de risco ou fatores de risco.

Por essa razão, a SBD sugere que sejam empregados os novos quesitos internacionais para investigação e diagnóstico da DMG, pois, mesmo que eles expressam deficiências, são os únicos que legitimamente demonstraram por estudo a combinação entre as taxas da glicemia da mãe e suas consequências na saúde do recém-nascido (SBD, 2014-2015), além de auxiliarem para constatar gestações de elevados riscos, na qual os resultados adversos podem ser evitados e vossas interferências são plausivelmente simples (COUSTAN, 2012).

Portanto, os novos critérios de diagnóstico da DMG adotados pela American Diabetes Association (ADA), oferecido pelo National Diabetes Data Group (IADPSG), outro pela Organização Mundial da Saúde e também pela Sociedade Brasileira de Endocrinologia e Metabologia. A Association recomenda o acompanhamento de grávidas até 25 anos de idade e entre a $24^{\underline{a}}$ e $28^{\underline{a}}$ semana de gestação, utilizando para averiguação o índice glicêmico de 1 hora após a ingestão de $50 \mathrm{~g}$ de dextrosol (SULLIVAN e MAHAN apud e NOGUEIRA, 2001).

Existem discussões entre os novos quesitos para diagnósticos e o quesito antigo, onde o ponto de corte era de $85 \mathrm{mg} / \mathrm{dL}$, pois em suas pesquisas, $13 \%$ das pacientes necessitaram de administração adicional de insulina para controle da glicemia que ainda não foram diagnosticadas por esses novos quesitos da IADPSG, o que os faz 
acreditar que os novos fundamentos podem interferir no não tratamento correto de algumas gestantes (SIMON; MARQUES; FARHAT, 2013; SACKS et al, 2012).

Queiros, Magalhães e Medina (2006) também ratifica que seja feita a prova de rastreio

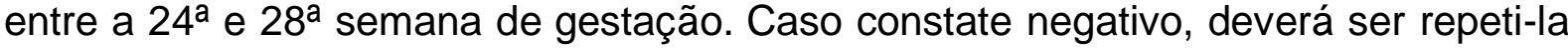
após a $32^{a}$ semana. As grávidas que, porventura, expressarem risco alto para a patologia devem fazer a prova preferivelmente logo após a revelação da gestação.

Caso o índice glicêmico de jejum seja superior ou igual a $126 \mathrm{mg} / \mathrm{dL}$, essa mulher já é diagnosticada portadora de DMG, visto que esse valor já qualifica, por si só, Diabetes Mellitus (QUEIROS; MAGALHÃES; MEDINA, 2006; SBD, 2014-2015). A Sociedade Brasileira de Diabetes preconiza que a taxa glicêmica de jejum deve ser requisitada na primeira consulta pré-natal, e, caso ela seja inferior a $92 \mathrm{mg} / \mathrm{dL}$, deve ser requisitado novamente o exame de taxa glicêmica de jejum no segundo trimestre da gravidez. (SBD 2014-2015).

Tendo desfecho negativo, elas prosseguem com o mesmo critério para as demais grávidas. Em todas as ocorrências de gestantes deve ser feita a Prova de Tolerância Oral à Glicose (PTOG), com 100g de glicose para certificação de DMG, com uma elaboração de jejum entre 10 a 14 horas e o colhimento deve ser feito na parte da manhã, em descanso. (QUEIROS; MAGALHÃES; MEDINA, 2006).

Depois de duas semanas de dieta, se os níveis glicêmicos permanecerem alterados, sendo em jejum um valor superior ou igual a $95 \mathrm{mg} / \mathrm{dL}$ e 1 hora pós-prandial igual ou superior a $140 \mathrm{mg} / \mathrm{dL}$, ou duas horas pós-prandiais encontram-se superior ou igual a $120 \mathrm{mg} / \mathrm{dL}$, torna-se indispensável iniciar o tratamento farmacológico.

\section{PRECAUÇÕES E UM TRATAMENTO POSSÍVEL}

Levando em consideração as possíveis desordens do DMG, para a mãe, assim como para o bebê, vislumbra-se a relevância de se argumentar mais sobre o assunto, substancialmente devido à mudança dos padrões de diagnóstico de DMG que ocorreu em 2005 e que se acham presentes até os dias atuais. 
É primordial que a paciente seja examinada para determinar se retornou ao estado de tolerância normal aos carboidratos e se a gravidez foi o marcador metabólico do diabetes. Examinando as literaturas, pôde-se vislumbrar a relevância da dieta, atividade física e controle glicêmico no tratamento, visto que os fatos sobre tratamento farmacológico ainda são insatisfatórios e questionáveis.

Quando se trata de tratamento medicamentoso, segundo Pontes et al. (2010), o cloridrato de metformina foi apontado o medicamento mais conveniente para grávidas com DMG, contudo, ainda hoje, há poucas literaturas que reafirmam a segurança e eficácia da mesma, mesmo que seus resultados associados à macrossomia do feto, peso ao nascer, internação em Unidade de Terapia Intensiva Neonatal, taxa hipoglicemia do recém-nascido, dentre outras desordens, sejam questionáveis.

A gestação requer certas demandas nutritivas que obrigam as substituições na alimentação. $O$ tratamento dietético adequado para uma gestante deve proporcionar uma nutrição satisfatória para a gestante quanto para o feto, objetivando uma quase euglicemia (BERTINI, 2001).

Embora não esteja comprovado que o exercício seja benéfico em mulheres portadoras com DMG, a alteração no estilo de vida pode perdurar logo depois do parto e auxiliar a precaver o começo da diabetes mellitus tipo 2 e suas desordens extemporâneas (CEYSENS et al., 2007).

A revisão de BRODY et al. (2003) aponta que um percentual de mais de $70 \%$ das mulheres com DMG desenvolvem hiperglicemia leve e seu tratamento se dá apenas com dieta. $\mathrm{O}$ auxílio do tratamento intensivo nessas mulheres ainda é incerto, mas nas mulheres com hiperglicemia elevada o tratamento com administração de insulina diminui a ocorrência de macrossomia. Apenas um percentual de $30 \%$ das gestantes com DMG fazem uso de insulina como forma de tratamento.

Segundo a Sociedade Brasileira de Diabetes (2014-2015), logo que o DMG é constatado, começa-se o tratamento não farmacológico, que se fundamenta em orientação alimentar e práticas de atividades físicas, desde que levadas em 
considerações as contraindicações obstétricas. A orientação alimentar objetiva o controle metabólico e elevação vultuosa de peso, sendo tolerado o ganho de 300 a $400 \mathrm{~g}$ por semana, a partir do segundo trimestre de gestação.

A dieta alimentar para as mulheres com DMG precisa compreender seis a sete refeições diárias, sendo três principais refeições, cerca de dois a três lanches e um antes de deitar. Ela precisa oferecer calorias e nutrientes o suficiente para as necessidades da gestação, bem como o alcance dos objetivos das taxas glicêmicas preliminarmente estabelecidos para que sejam dispensados casos de hiperglicemia, hipoglicemia ou de cetose. (QUEIROS; MAGALHÃES; MEDINA, 2006).

A elevação do peso diversifica conforme com o IMC de cada gestante. Para mulheres com o IMC superior a 19,8 kg/m², a elevação média de peso é entre 12,5 a $18 \mathrm{~kg}$; IMC entre 19,8 e $26 \mathrm{~kg} / \mathrm{m}^{2}$, elevação média de 11 a $16 \mathrm{~kg}$; IMC entre 26,1 a $29 \mathrm{~kg} / \mathrm{m}^{2}$, elevação média de 7 a $11 \mathrm{~kg}$; IMC elevação que $29 \mathrm{~kg} / \mathrm{m}^{2}$, elevação de menos de 6 kg (QUEIROS; MAGALHÃES; MEDINA, 2006).

A prática de exercícios físicos tem como objetivo reduzir a intolerância à glicose por meio da restrição cardiovascular que produz elevação da afinidade e da ligação da insulina ao seu receptor por meio da redução da gordura intra-abdominal, elevação dos transportadores de glicose sensíveis à insulina no músculo, elevação do fluxo sanguíneo em tecidos sensíveis à insulina e redução dos níveis de ácidos graxos livres.

A grande preocupação é a segurança da gestação para a mãe e para o feto, os critérios mais importantes a serem avaliados durante a prática esportiva é o bemestar materno-fetal, como: frequência cardíaca e frequência cardíaca fetal, pressão arterial, temperatura e dinâmica uterino-maternas.

Vários tipos de aparelhos e de atividades físicas foram prescritos para as gestantes com DMG, tais como: bicicleta ergométrica vertical, remo, bicicleta ergométrica horizontal (com encosto) e ergométrica para membros superiores. 
De maneira geral, a insulina somente é introduzida como remédio, quando a dieta e os exercícios não conseguiram atingir um controle metabólico adequado. A insulina foi isoladamente utilizada e incialmente prescritas nos casos de diabetes. Entre os diversos tipos de insulina hoje disponíveis temos a de curta ação (lispro e regular) e as de longa ação a lenta e a ultralenta.

Weinertet et al. (2011) indica a glibenclamida como o medicamento de preferência entre as sulfonilureias para gestantes, apontada como segura e eficaz a começar do segundo trimestre de gestação. Silva et al. (2007), em suas análises, concluiu que a classe de gestantes que exibiram maiores resultados com a administração de glibenclamida foi as com período da gestação superior a 19 semanas e elevação de peso de, no máximo até $9 \mathrm{~kg}$, além de não ser notado comprometimento dos bebês, situação que reforça a segurança de sua administração.

No entanto, há uma discordância em relação à segurança da glibenclamida, quando declaram que a própria não é aprovada pelo Food and Drug Administration (FDA), sendo necessárias mais pesquisas sobre esse medicamento. Em pesquisas feitas com animais, os medicamentos acarbose, glibenclamida e insulina são nomeados como classe B para utilização no período da gestação, ou seja, não exibiram efeitos maléficos fetais, não havendo pesquisas científicas que confirmem efeitos nocivos destes medicamentos em humanos (LARIMORE; PETRIE, 2000; apud SILVA et al., 2005).

As doses de insulina recomendadas são administradas conforme o controle de glicemia da gestante elevando-se a insulina regular quando ocorre a insulina NPH levando em consideração as glicemias de jejuns e as pré-prandiais.

O tratamento insulinoterápico para DMG é adotado quando o controle da glicemia não for eficaz, é recomendado a administração de insulina, com associação de dieta e exercícios físicos. Porém, não há um protocolo padrão de orientação no que se referem as diferentes dosagens para as diferentes fases da gestação, pois elas mudam de acordo com a variação do peso (BASSO et al., 2007). 
De acordo com Detschet al (2012), a razão primordial para a administração de insulina foi a junção entre DMG prévio e causas de risco, como histórico familiar de DM em parentes de primeiro grau. As principais causas de risco para o DMG são: identificação do DMG tardia, idade superior a 30 anos, polidrâmnio, crescimento excessivo do feto, identificação do DMG tardio, começo atrasado do acompanhamento pré-natal, malformações do feto e morte do feto ou natimortos, obesidade ou sobrepeso, baixa estatura, acúmulo de adiposidade abdominal, baixa renda da família, antecedentes obstétricos de abortos de repetição, crescimento excessivo do feto, ovário policístico, crescimento excessivo do feto, macrossomia, hipertensão arterial ou pré-eclâmpsia na gravidez atual (COSTA et al., 2015; SBD 2014-2015; DETSCH et al., 2012).

\section{CONCLUSÕES}

O grandioso impacto do descobrimento da insulina, o desmedido conhecimento do dispositivo de acometimento fetal pela hiperglicemia e dos critérios terapêuticos no diabetes mellitus gestacional são de indiscutíveis relevâncias. Por consequência a acentuada procura por mecanismos optativos e alcançáveis a toda população são de suma importância e devem ser valorizados.

Em síntese, o DMG é uma patologia que afeta mulheres no período da gravidez. No decorrer da gestação, nas grávidas predispostas poderão desenvolver complicações no metabolismo podendo resultar em diabetes mellitus gestacional e sua predominância varia de 3\% a 13\% do total de gestantes, diversificando de acordo com a população analisada e o critério empregue. (SBD, 2014-2015).

Assim, o DMG passou a ser considerado como um tormento de saúde pública, e mesmo quando há um pré-natal bem acompanhado, podem perdurar alguns problemas como o crescimento do feto e outros desiquilíbrios. Desta forma, a gestante precisará fazer um bom pré-natal e passará a se conscientizar e ficar atenta aos diagnósticos e à fisiopatologia, bem como ao tratamento e as possíveis complicações e aos riscos que o feto poderá correr. Tais cuidados são de suma importância para uma gravidez segura e sem problemas para a mamãe e ao bebê. 
Nesse entendimento, a Organização Mundial da Saúde (OMS) preconiza que um bom acompanhamento da DMG contenha dieta, controle metabólico satisfatório, exercícios físicos e medicação, além do pré-natal feito por uma equipe multiprofissional especialista (WHO, 2013).

Este estudo teve como alvo principal argumentar e ponderar sobre o DMG tendo uma visão geral sobre os cuidados, as possíveis intervenções, e complicações durante a gravidez. Desta forma, estudo teve como metodologia descritiva e exploratória, de revisão bibliográfica, empregando para isso, os seguintes descritores: DMG, Fatores de Risco, Cuidados. A pesquisa compreendeu o período de 2005 a 2018, por abordar do que existe de mais recente na literatura científica sobre Diabetes Mellitus Gestacional.

\section{REFERÊNCIAS}

ACM. Manual de pesquisa das Diretrizes do ACSM para testes de esforço e sua prescrição. 4ª edição Rio de Janeiro: Guanabara Koogan, 2003, p.280-283.

American Diabetes Association. Diagnosis and classification of diabetes mellitus. Diabetes Care 2011 Jan; 34 Suppl 1:S62-9. 2. IDF Clinical Guidelines Task Force. Global Guideline on Pregnancy and Diabetes. Brussels: International Diabetes Federation, 2009.

American Diabetes Association. Diagnosis and classification of diabetes mellitus. Diabetes Care 2010 Jan; 33 Suppl 1: S62-9. 47.

BASSO N. A. S. Et al. Insulinoterapia, controle glicêmico materno e prognóstico - perinatal - diferença entre o diabetes gestacional e o clínico. Revista Brasileira de Ginecologia e Obstetrícia. Rio de Janeiro, v. 5, p.254-259, 2007.

BERTINI, A. M. Diabetes mellitus. In: GUARIENTO, A. MAMEDE, J. A. V. Medicina materno-fetal. São Paulo: Atheneu, 2001. 
BRASIL. Ministério da Saúde. Secretaria de Atenção à Saúde. Departamento de Ações Programáticas Estratégicas. Gestação de alto risco: manual técnico / Ministério da Saúde, Secretaria de Atenção à Saúde, Departamento de Ações Programáticas Estratégicas. 5를 ed. Brasília: Editora do Ministério da Saúde, 2010. p 302.

BRODY SC, Harris R., Lohr K. Screening for gestational diabetes: a summary of the evidence for the U.S. Preventive Services Task Force. Obstet Gynecol. 2003; 101:38092.

CEYSSENS P.J., Mesyanzhinov V., Sykilinda N., Briers Y., Roucourt B., Lavigne R., Robben J., Domashin A., Miroshnikov K., Volckaert G., Hertveldt K. The genome and structural proteome of YuA, a new Pseudomonas aeruginosa phage resembling M6. J. Bacteriol. 2008; 190:1429-1435

COSTA R. C. Et al. Diabetes Gestacional assistida: perfil e conhecimento das gestantes. Revista Saúde (Santa Maria), v. 41, n. 1, p. 131 - 140, 2015.

COUSTAN D. R.The American Diabetes Association and the International Association of Diabetes and Pregnancy Study Groups Recommendations for Diagnosing Gestational Diabetes Should Be Used Worldwide. ClinicalChemistry. V. 58, n. 7, p. 1094-1097, 2012.

DETSCH J. C. M. et al. Marcadores para o diagnóstico e tratamento de 924 gestações com diabetes melito gestacional. Arquivos Brasileiros de Endocrinologia e Metabolismo. São Paulo, v. 55, n. 6,2011.

FERRARA A. Increasing prevalence of gestational diabetes mellitus: a public health perspective. Diabetes Care. 2007; 30, Suppl 2:S141-6.

IDF Diabetes Atlas. Seventh Edition ed. Brussels: International Diabetes Federation; 2015. Disponível em: http://www.diabetesatlas.org 2. Pesquisa Nacional de Saúde 2013: percepção do estado de saúde, estilos de vida e doenças crônicas. Brasília: Instituto Brasileiro de Geografia e Estatística; 2014. Disponível em: ftp://ftp.ibge.gov.br/ PNS/2013/pns2013.pdf 3. 
Instituto Brasileiro de Geografia e Estatística. Disponível em: http://ibge.gov.br/home/presidencia/noticias/noticia_visualiza. php?id_noticia=1699\&id_pagina=1. Acessado em 28/junho/2019.

KITZMILLE, J. L.; DAVIDSON, M. B. Diabetes e gravidez. In: DAVIDSON, M. B. Diabetes Mellitus: diagnóstico e tratamento. Rio de Janeiro: Revinterr, 2001. P. 277-303.

NOGUEIRA, A. I. Diabetes mellitus e gravidez. Rio de Janeiro: MEDSI, 2001. P. 465-568-644.

O'SULLIVAN JB, Mahan CM. Criteria for the Oral Glucose Tolerance Test in Pregnancy. Diabetes. 1964; 13:278-85. 18.

OMS. Obesidade Prevenindo e Controlando a Epidemia Global. 1aㅡ ed. São Paulo: Roca; 2004. p. 256. 36. Bolognani CV, Souza SS, Dias A, Rudge MVC, Calderon IMP. Circunferência da cintura na predição do diabetes mellitus gestacional. 2011 [em publicação].

ORGANIZAÇÃO PAN-AMERICANA DA SAÚDE 20. Carpenter MW, Coustan DR. Criteria for screening tests for gestational diabetes. Am J Obstet Gynecol. 1982; 1; 144(7):768-73. 21. Proceedings of the Third International Workshop-Conference on Gestational Diabetes Mellitus; 1990 November 8-10; Chicago, Illinois; 1990.

QUEIROS J, MAGALHÃES A., MEDINA J. L. Diabetes gestacional: uma doença, duas gerações, vários problemas. Revista Brasileira de Endocrinologia, Diabetes e Metabolismo, v. 1, n. 2, p. 19-24, 2006.

SANCOVSKI, M. Diabetes e gravidez. Terapêutica em diabetes. Centro BD de educação em diabetes. V. 5, n. 25, p. 1-5, 1999.

SILVA J. C.et al. Resultados preliminares do uso de anti-hiperglicemiantes orais no diabete Melito gestacional. Revista Brasileira de Ginecologia e Obstetrícia. V. 27, n.8, p. 461-466, 2005. 
SILVA J. C.et al. Tratamento do diabetes mellitus gestacional com glibenclamida - fatores de sucesso e resultados perinatais. Revista Brasileira de Ginecologia e Obstetrícia. Rio de Janeiro. V. 29, n. 11, p. 555-560, 2007.

SIMON C. Y., MARQUES M. C. C., FARHAT H. L. Glicemia de jejum do primeiro trimestre e fatores de risco de gestantes com diagnostico de Diabetes Melito gestacional. Revista Brasileira de Ginecologia e Obstetrícia. V. 35, n. 11, 2013.

SOCIEDADE BRASILEIRA DE DIABETES. DIABETES MELLITUS GESTACIONAL: DIAGNÓSTICO, TRATAMENTO E ACOMPANHAMENTO PÓS-GESTAÇÃO. In: Diretrizes da Sociedade Brasileira de Diabetes: 2014- 2015/Sociedade Brasileira de Diabetes; [organização Jose Egidio Paulo de Oliveira, SergioVencio]. - São Paulo: AC Farmacêutica, 2015.

TOMBINI, M. Guia completo sobre Diabetes da American Diabetes Association. Rio de Janeiro: Anima, 2002. p. 44-45-340-341.

WEINERT LS et al. Diabetes gestacional: um algoritmo de tratamento multidisciplinar. Arquivos Brasileiros de Endocrinologia Metabólica. V. 55, n. 7, p. 435-445, 2011.

WHO expert Committe on Diabetes Mellitus Technical Report: World Health Organization 1964. Report No.: 310. 19. Classification and diagnosis of diabetes mellitus and other categories of glucose intolerance. National Diabetes Data Group. Diabetes. 1979; 28(12): 1039-57.

Enviado: Maio, 2020.

Aprovado: Novembro, 2020. 